\title{
Analytical Modeling of Ad Hoc Cognitive Radio Environment for Optimum Power Control
}

\author{
Indu Bala \\ ECE Department \\ Punjabi University \\ Patiala, India
}

\author{
Manjit Singh Bhamrah \\ ECE Department \\ Punjabi University \\ Patiala, India
}

\author{
Ghanshyam Singh \\ ECE Department \\ JUIT Solan \\ H. P., India
}

\begin{abstract}
Cognitive Radio is an emerging wireless technology that has grabbed the attention of researchers, academicians, and telecommunication industries worldwide. Recent survey reports show that unlicensed frequency bands have become overcrowded from past few years with widespread acceptance of diverse wireless technology applications whereas, licensed frequency bands are being used intermittently. To deal with the conflicts between unlicensed spectrum overuse and underutilization of licensed frequency band, Cognitive Radio technology has been evolved eventually. It allows unlicensed subscribers to access licensed subscriber frequency band with some power constraints such that minimum or no interference should be experienced by primary user and its quality of service should not be affected while sharing its spectrum with unlicensed secondary subscriber. In this paper, distance based power control scheme has been analyzed for overlay ad-hoc cognitive radio network. It is shown that algorithm provides optimum power control as long as the separation between cognitive transmitter and receiver is greater than or equals to maximum decodable radius of primary transmitter.
\end{abstract}

\section{Keywords}

Federal communication commission (FCC), Primary user (PU), secondary User (SU), Quality of services (QoS), Cognitive Radio (CR).

\section{INTRODUCTION}

Electromagnetic spectrum is precious and very limited natural resource therefore its efficient use is of utmost importance. From past few years, we all have seen tremendous growth in wireless technology applications ranging from remote controlled kid's toy car, home automation system, mobile phones to NASA's space shuttle program. As a matter of fact, the demand for more bandwidth has overgrown.

The use of radio spectrum in each country is nationally governed by the corresponding government agencies like Federal Communications Commission (FCC) which auction the spectra to the licensed holders known as primary users, on a long term basis over a large geographical region. This is known as fixed assignment policy. The major bottleneck with this policy is that it does not ensure that the bands are being utilized efficiently all the time or not [1].

Recent report by FCC has revealed that a large portion of the assigned spectrum is used sporadically. The temporal and geographical variations in the utilization of the assigned spectrum range from $15 \%$ to $85 \%$. For example, the utilization in the $3-4 \mathrm{GHz}$ frequency band is $0.25 \%$ and drops to $0.13 \%$ in the $4-5 \mathrm{GHz}$ band. Even in the most crowded bands, such as below $2 \mathrm{GHz}$, the utilization is less than $50 \%$. To deal with conflicts between spectrum overcrowding and spectrum under utilization, J. Mitola proposed a spectrum agile Cognitive Radio technology that allows unlicensed users (secondary user) to have an access of the licensed users (Primary User) spectrum opportunistically. Many schemes have been proposed by researchers for the spectrum sharing by both types of users. In this paper, overlay scheme has been used in which both secondary users (SU) are allowed to share the licensed frequency band of primary user (PU) simultaneously, provided

(i) Interference from SU to PU is negligible or below threshold value.

(ii) QoS of PU is not affected by SU.

To minimize the interference from SU to PU and in order to have guaranteed QoS to the PU, transmitted power of SU must be controlled intelligently based on its distance from licensed primary user. Many researchers have used sensing information as a proxy to calculate distance between primary and secondary users, but, in this paper sensing free power control technique has been analyzed from geometric point of view.

The rest of the paper is organized as follows. In Section II, system model, assumptions and formulation of power control problem is done. Section III gives mathematical modeling and flow diagram of SU's power control. System parameters and simulation results are presented in section IV, followed by conclusion in section $\mathrm{V}$.

\section{SYSTEM MODELING \& PROBLEM FORMULATION}

The system model is proposed for overlay ad-hoc network. Primary base station is using licensed TV band for transmission. The location of primary and secondary receivers is define by their polar co-ordinates i.e. $(\mathrm{dpp}, \theta 1)$ and $(\mathrm{dpc}, \theta 2$ ) respectively[2]. Both, Primary transmitter and receiver, are assumed fixed and all angular positions are measured with respect to the reference axis as shown in Fig.1.The blue colored triangles are showing primary transmitter and receiver whereas, red diamond shapes are representing cognitive transmitter and receiver. Cognitive users are trying to have an access of the licensed band of primary user opportunistically without offering significant interference and without quality degradation of primary user's services [3]. The spectrum sharing scenario and system model is shown in Fig. 1.The notations used in power calculations are given in Table 1.

Using two ray ground propagation model, the received power $\mathrm{Pr}$ is given by

$P_{r}=\frac{P_{t} G_{t} G_{r} h_{t}^{2} h_{r}^{2}}{d^{\alpha}}$ 
Where, Pt is transmitting power, Gt and Gr are transmitting and receiving antenna gain respectively. ht and $\mathrm{hr}$ are transmitting and receiving antenna heights and $\mathrm{d}$ is spacing between transmitter and receiver and $\alpha$ is the path loss factor.

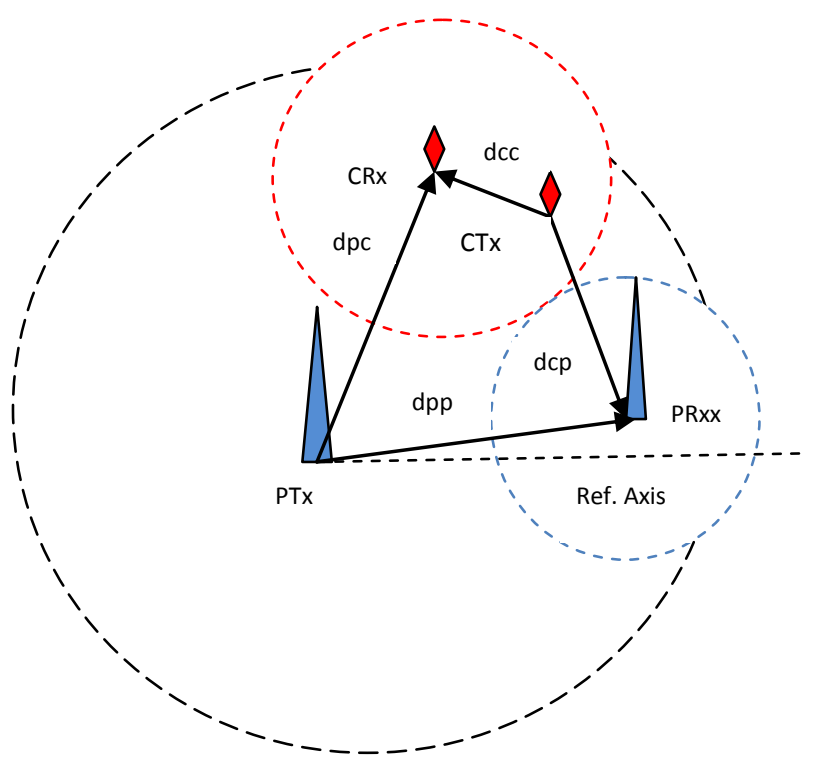

Fig.1: System Model

Table 1: Notations

\begin{tabular}{|c|l|}
\hline Symbol & \multicolumn{1}{|c|}{ Parameter } \\
\hline $\mathrm{dpp}$ & Distance between primary Tx and Rx \\
\hline $\mathrm{dpc}$ & Distance between primary Tx and cognitive Rx \\
\hline $\mathrm{dcp}$ & $\begin{array}{l}\text { Distance Between primary Tx \& cognitive } \\
\text { receiver Rx }\end{array}$ \\
\hline $\mathrm{dcp}$ & Distance between Cognitive Tx \& primary Rx. \\
\hline $\mathrm{dcc}$ & Distance between Cognitive Tx and Rx. \\
\hline $\mathrm{Pb}$ & Transmitted power of primary Tx \\
\hline $\mathrm{Pc}$ & Transmitted power of Cognitive Tx \\
\hline$\alpha$ & Path loss factor \\
\hline$\theta p c$ & $\begin{array}{l}\text { Relative angle between primary and cognitive } \\
\text { Rxs. }\end{array}$ \\
\hline $\mathrm{Thp}$ & Threshold value of Primary user \\
\hline $\mathrm{Thc}$ & Threshold value of Cognitive user \\
\hline $\mathrm{SIRp}$ & Signal to interference ratio for primary Rx \\
\hline $\mathrm{SIRc}$ & Signal to interference ratio for Cognitive Rx \\
\hline Popt & Optimum transmission power for Cognitive Tx \\
\hline Pmax & Maximum power of Cognitive Tx \\
\hline Pmin & Minimum power of Cognitive Tx \\
\hline $\mathrm{Rd}$ & Maximum decodable radius of Primary CTx. \\
\hline$\delta$ & $\begin{array}{l}\text { Ration of transmitting powers of primary and } \\
\text { cognitive user. }\end{array}$ \\
\hline
\end{tabular}

The distance between primary and cognitive receiver can be computed geometrically by using law of cosine and will be given by

$d_{p c}=\sqrt[n]{d_{p p}+d_{p c}-2 d_{p p} d_{p c} \cos \theta_{p c}}$

In overlay transmission scheme, both primary and cognitive users are allowed to transmit simultaneously provided interference offered from cognitive transmitter to the legacy primary user is least and QoS of primary user should not be degraded with the presence of cognitive user [4]. Keeping these objectives in mind, the problem could be formulated as maximize Area of simultaneous transmission with constraints $\left(\begin{array}{c}S I R_{p}>T h_{p} \& S I R_{c}>T h_{c} \\ P_{\min } \leq P_{\text {opt }} \leq P_{\max }\end{array}\right)$

III. Mathematical Modelling \& Flow Diagram

Using these constraints various distance may be calculated geometrically and are given below [5]

$d_{c c}<d_{p c}\left(\frac{\delta}{T h_{c}}\right)^{\frac{1}{\alpha}}$

$d_{c p}>d_{p p}\left(\delta . T h_{p}\right)^{\frac{1}{\alpha}}$

The transmission power calculation can be done by knowing distances and can be formulated as

$\left(\begin{array}{ll}d_{c p}+d_{c c}=d_{p c} & ; P_{t}=P_{o p t} \\ d_{c p}+d_{c c}>d_{p c} & ; P_{t}=P_{\min } \\ d_{c p}+d_{c c}<d_{p c} & ; P_{t}=P_{\max }\end{array}\right)$

The flow diagram of power control scheme is as shown in Fig.2

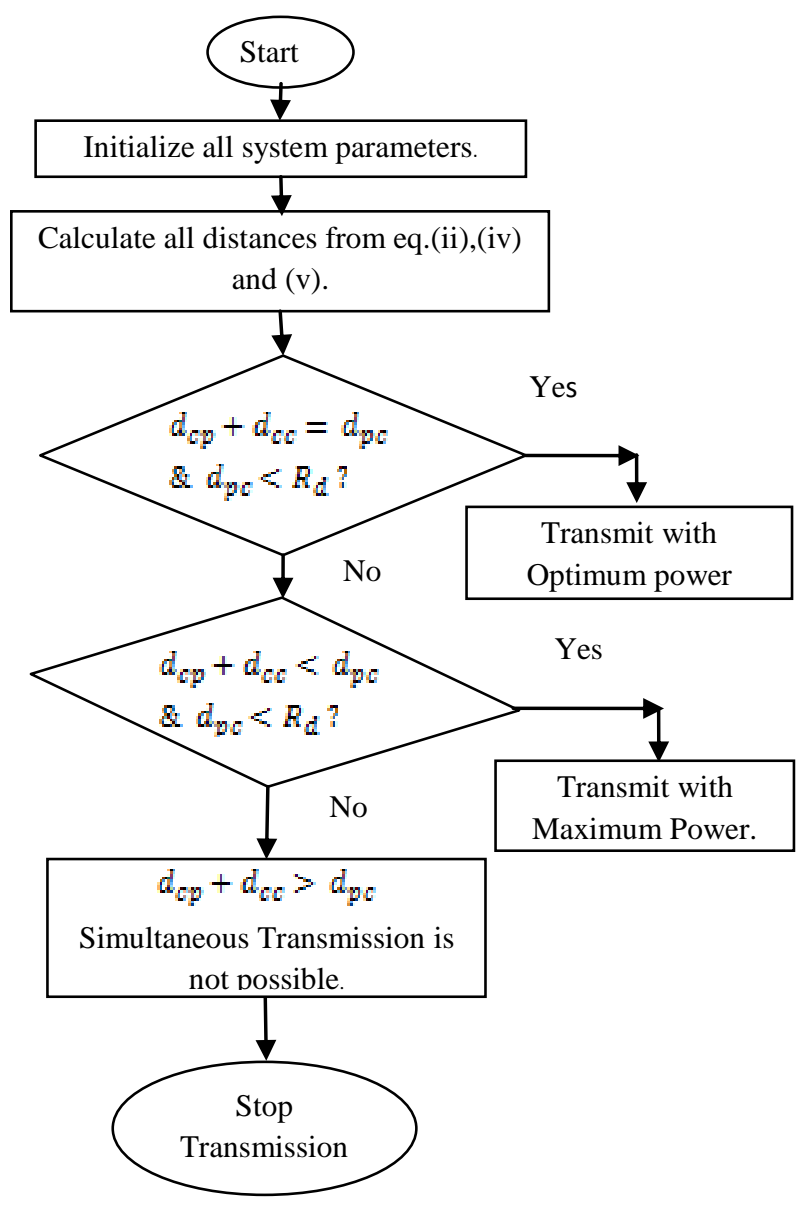

Fig.2: Flow Chart 


\section{RESULTS \& DISCUSSIONS}

In this section, simulation parameters and simulated results are given. All the results have been simulated in MATLAB 7.1. The parameters used in simulations are enlisted in Table 2.

Table 2. Simulation Parameters

\begin{tabular}{|l|l|l|}
\hline S.No. & \multicolumn{1}{|c|}{ Parameter } & \multicolumn{1}{c|}{ Value } \\
\hline 1. & Primary Transmission Power & $80 \mathrm{dBm}$ \\
\hline 2. & Minimum CR Tx Power & $30 \mathrm{dBm}$ \\
\hline 3. & Maximum CR Tx Power & $50 \mathrm{dBm}$ \\
\hline 4. & Location of PR Rx & $\left(50 \mathrm{~km}, 0^{0}\right)$ \\
\hline 5. & Location of CR Rx & $\left(50 \mathrm{~km}, 60^{\circ}\right)$ \\
\hline 6. & Threshold value of PR Rx & $30 \mathrm{~dB}$ \\
\hline 7. & Threshold value of CR Rx & $3 \mathrm{~dB}$ \\
\hline 8. & Propagation path loss factor & 3 \\
\hline
\end{tabular}

Fig. 3 is showing the performance of power control algorithm with respect to the separation between primary transmitter and cognitive receiver. Fig.3 and Fig.4 are showing that the optimum transmission power for cognitive user for angular spacing from $0^{\circ}$ to $90^{\circ}$. For the range up to $80^{\circ}$ the optimum transmission power is less than maximum transmission power i.e. 100 watt and at $90^{\circ}$ optimum transmission power become equals to the maximum value of 100 watts.

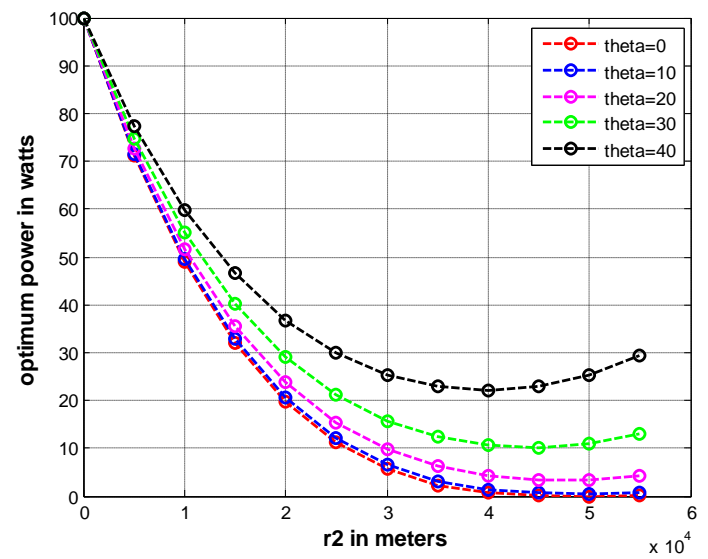

Fig.3: optimum power v/s dpc with angular placement ( 0 to 40 degrees)

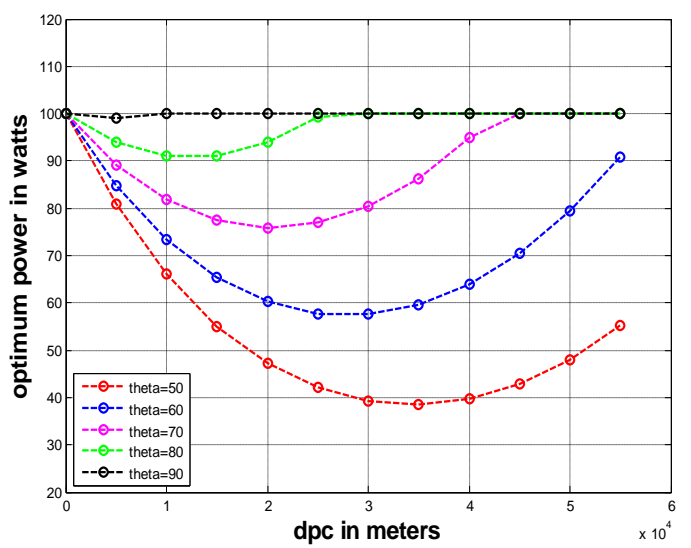

Fig.4: optimum power v/s dpc with angular placement (50to 90 degrees)

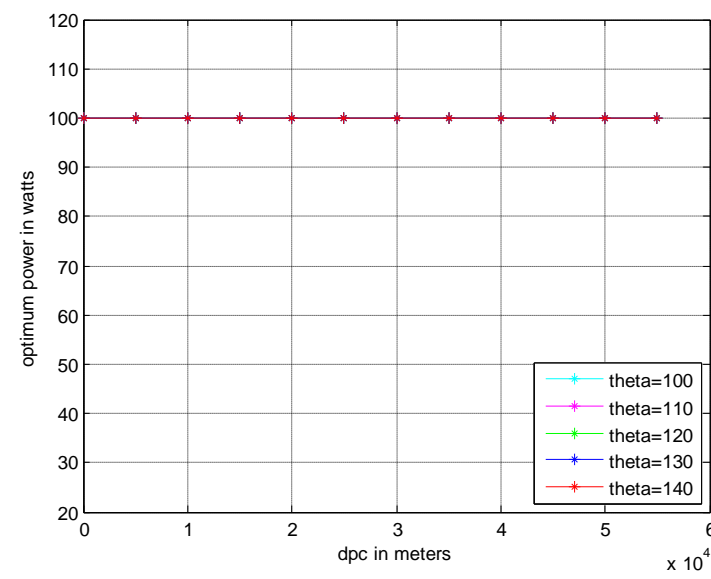

Fig.5: optimum power v/s dpc with angular placement (100to 140 degrees)

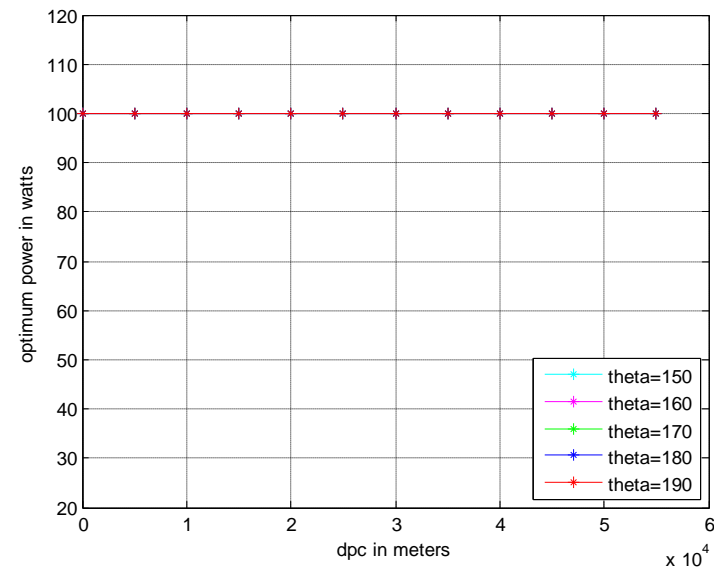

Fig.6: optimum power v/s dpc with angular placement (150to 190 degrees)

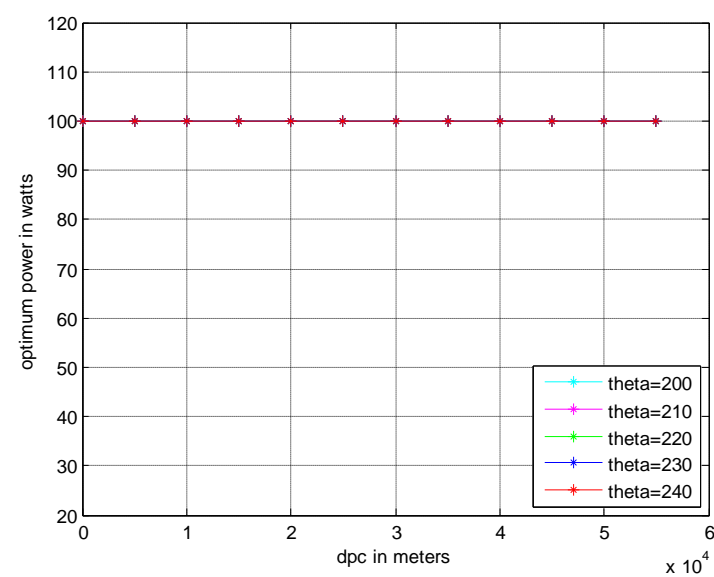

Fig.7: optimum power v/s dpc with angular placement (200to 240 degrees)

Fig.5 to Fig. 8 is showing that from a range $100^{\circ}$ to $270^{\circ}$ cognitive user can transmit with maximum power without any interference to primary user. Fig. 8 and fig 9 showing optimum transmission power for a range $280^{\circ}$ to $340^{\circ}$ with respect to the separation between primary transmitter and cognitive receiver. 


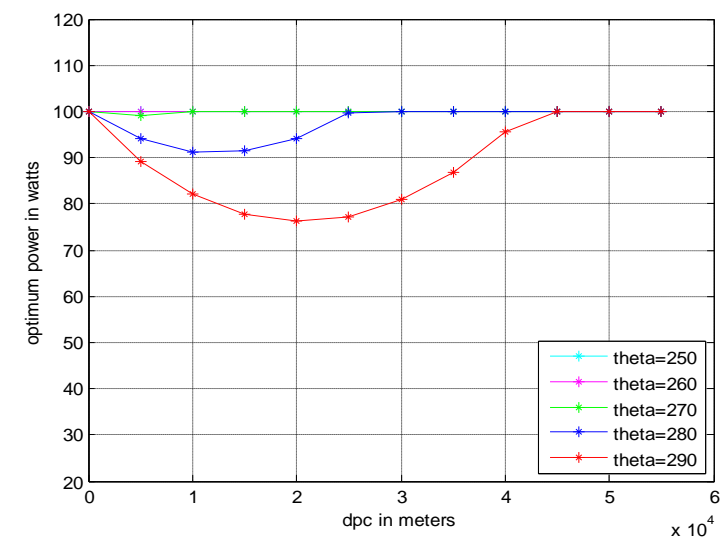

Fig.8: optimum power v/s dpc with angular placement (250 to 290 degrees)

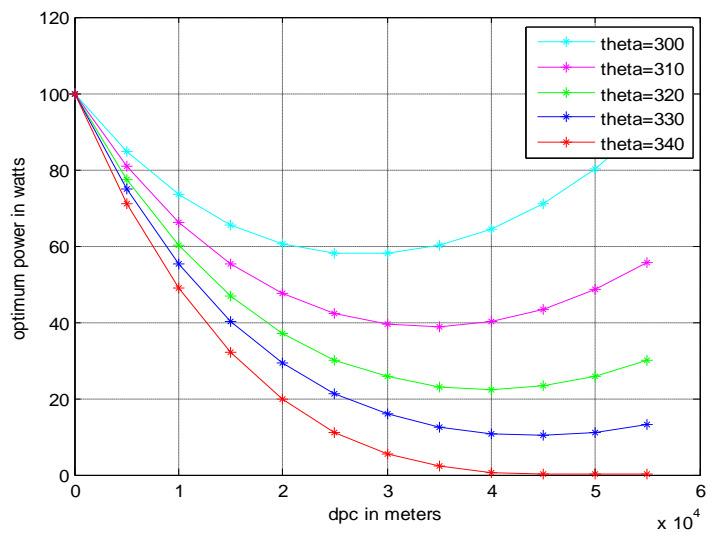

Fig.9: optimum power v/s dpc with angular placement (300 to 340 degrees)

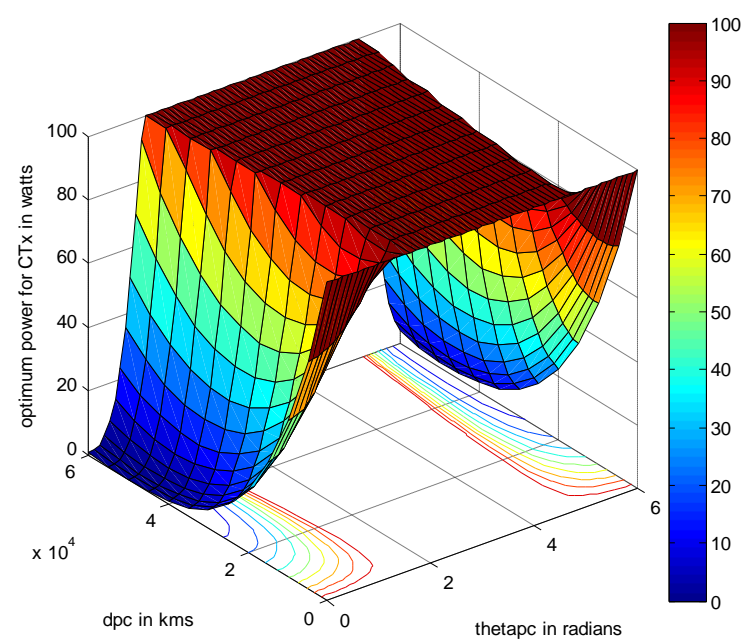

Fig.10: Plane of optimum power of CTx

Fig. 10 is showing plane of optimum power of cognitive transmitter for simultaneous spectrum sharing with primary user [6]. Again, it is clear from Fig.10 that optimum power remains constant i.e. maximum 100 watts for certain range of $\theta_{\mathrm{pc}}$. The reason for restricting the optimum power to its maximum value is that the separation between primary transmitter and cognitive receiver is far greater than from its maximum decodable radius i.e. dcc for specific $\theta_{\mathrm{pc}}$. Therefore, even if the CRs transmitter power is maximum, it will not disrupt the operation of primary user. Similarly, optimum power for the transmission is approaching to zero for certain range of $\theta_{\mathrm{pc}}$ as shown in Fig. 3 and Fig.9. This is due to the reason that separation between CR transmitter and receiver is far less than minimum required separation distance and, therefore, CR transmitter is lying within decidable range of primary. Therefore, Cognitive transmitter must be quite with in this area i.e. should not make any transmission. Thus, the optimum power control for cognitive user can only be achieved if and only if the separation between cognitive transmitter and receiver is significant and less than maximum decodable radius of the secondary cognitive transmitter.

\section{CONCLUSION}

In this paper, optimum power control algorithm is discussed for the simultaneous access of the licensed user's frequency band by cognitive user. The results have been simulated for overlay transmission scheme in which both primary as well cognitive users can share the spectra simultaneously with some constraints over cognitive user's transmission power. For given system parameters performance of geometrical distance based power control algorithm is evaluated. It is shown that algorithm works well and provide optimum power control as long as the separation between cognitive transmitter and receiver is greater than or equals to maximum decodable radius of primary transmitter.

\section{REFERENCES}

[1] I.F.Akyildiz, W.Y. Lee, M.C. Varun and S. Mohanty, "Next generation/dynamic spectrum access/cognitive radio wireless networks: survey," computer networks, Vol. 50, pp. 2127-2159, Sept. 2006.

[2] S. M. Hur, S. Mao, Y.T. Hou,K. Nam and J.H.Reed, "Exploting location information for concurrent transmissions in multihop wireless networks," IEEE Transactions on Vehicular Technology, Vol.58, Issue no. 1, pp. 314- 323, Jan. 2009.

[3] L.Qian, X. Li, J. Hwu, "Secondary Transmission power of cognitive radios for dynamic spectrum access," in the proceedings of Chinacom, pp. 7-12, 2007.

[4] Samuel Montejo Sanchez, RichardDemo Souza, Evelio M.G. Fernandez and Vitalio Alfonso Reguera, "Rate and energy efficient power control in a Cognitive radio adhoc networks," in IEEE signal processing letters, Vol. 20, No.5, May 2013.

[5] Samuel Montejo Sanchez, RichardDemo Souza, Evelio M.G. Fernandez and Vitalio Alfonso Reguera, "Impact of rate control on the performance of a cognitive radio ad-hoc network," in IEEE communication letters, Vol. 16, issue no.9, Sept. 2012.

[6] Yi Song,Jiang (Linda)Xie, "Optimal power control for concurrent Transmission of location aware mobile cognitive radio Ad hoc Network" in the conference proceedings of IEEE Global Telecommunications Conference, pp 1-6, Dec.2009. 